\title{
OPERATOR-VALUED FREE MULTIPLICATIVE CONVOLUTION: ANALYTIC SUBORDINATION THEORY AND APPLICATIONS TO RANDOM MATRIX THEORY
}

\author{
SERBAN T. BELINSCHI, ROLAND SPEICHER, JOHN TREILHARD, \\ AND CARLOS VARGAS
}

\begin{abstract}
We give an explicit description, via analytic subordination, of free multiplicative convolution of operator-valued distributions. In particular, the subordination function is obtained from an iteration process. This algorithm is easily numerically implementable. We present two concrete applications of our method: the product of two free operator-valued semicircular elements and the calculation of the distribution of $d c d+d^{2} c d^{2}$ for scalar-valued $c$ and $d$, which are free. Comparision between the solution obtained by our methods and simulations of random matrices shows excellent agreement.
\end{abstract}

\section{INTRODUCTION}

Free probability is a quite recent theory that has gained interest in the last few years. One of its main applications is on the field of random matrices. More specifically, it provides a conceptual way of understanding the distribution of the eigenvalues of several large random matrices. The variety of matrix models where free probability can be used is growing in accordance to the developments of the theory.

The crucial requirement that allows the treatment of a random matrix model with the algebraic and analytical machinery of free probability is that the matrices involved should satisfy (asymptotically, as their size tends to infinity) freeness relations. Some of the most important random matrices, such as Wigner and Haar Unitary matrices, were shown, starting with the basic work [27, to have these freeness requirements among themselves and with respect to deterministic matrices.

The applicability of free probability increased rapidly, in different directions, with the implementation of Voiculescu's operator-valued version of the theory. The main idea is that operator-valued freeness is a much less restrictive condition, but still most of the features of usual free probability theory are present. We are now able to work with block Gaussian matrices [18, 17, rectangular random matrices of different sizes [5] and more complicated combinations of random and deterministic matrices [21], where scalar-valued freeness breaks up.

Date: November 1, 2018.

Work of S. Belinschi was supported by a Discovery Grant from NSERC. S. Belinschi also gratefully acknowledges the support of the Alexander von Humboldt foundation and the hospitality of the Free Probability research group at the Universität des Saarlandes during the work on this paper.

Work of J. Treilhard and C. Vargas was supported by funds from R. Speicher from the Alfried Krupp von Bohlen und Halbach Stiftung ("Rückkehr deutscher Wissenschaftler aus dem Ausland") and from the DFG (SP 419/8-1), respectively. 
Whereas the analytic theory of scalar-valued free convolutions is far evolved (and thus we have now a variety of ways to deal with asymptotic eigenvalue distributions of matrices which are free), the same cannot be said about the status of operatorvalued convolutions. In particular, at the moment we do not have an analytic description of operator-valued convolutions which would be easily and controllably implementable on a computer. Thus, numerical investigations for the above mentioned random matrix models were done in a more or less ad hoc way. Our main aim is to improve on this situation and provide a coherent analytic description of operator-valued free convolution and show its usefulness for dealing with random matrix questions. In the present paper we concentrate on multiplicative free convolution, the question of additive free convolution will be addressed in another paper [3].

The present paper is motivated by the following problem (which was communicated to us by Aris Moustakas in this form in the context of wireless communications and, independently, by Raj Rao for the special case $b_{1}=b_{2}$ and $a_{1}=a_{2}^{2}$ in the context of random graphs): If $\left\{a_{1}, a_{2}\right\}$ and $\left\{b_{1}, b_{2}\right\}$ are free, then it is not true in general that the elements $a_{1} b_{1} a_{1}, a_{2} b_{2} a_{2}$ are free. This has made the distribution of $c=a_{1} b_{1} a_{1}+a_{2} b_{2} a_{2}$ quite inaccessible up to now.

We observe, however, that the distribution of $c$ is the same (modulo a Dirac mass at zero) as the distribution of the element

$$
\left(\begin{array}{cc}
a_{1} b_{1} a_{1}+a_{2} b_{2} a_{2} & 0 \\
0 & 0
\end{array}\right)=\left(\begin{array}{cc}
a_{1} & a_{2} \\
0 & 0
\end{array}\right)\left(\begin{array}{cc}
b_{1} & 0 \\
0 & b_{2}
\end{array}\right)\left(\begin{array}{cc}
a_{1} & 0 \\
a_{2} & 0
\end{array}\right),
$$

which in turn has the same moments as

$$
\left(\begin{array}{cc}
a_{1}^{2} & a_{1} a_{2} \\
a_{2} a_{1} & a_{2}^{2}
\end{array}\right)\left(\begin{array}{cc}
b_{1} & 0 \\
0 & b_{2}
\end{array}\right)=: A B
$$

The advantage of this reformulation is that the matrices $A$ and $B$ are free with amalgamation over the algebra $M_{2}(\mathbb{C})$ of $2 \times 2$ constant matrices. Hence, the distribution that we are looking for will be given by first calculating the $M_{2}(\mathbb{C})$-valued free multiplicative convolution of $A$ and $B$ to obtain the $M_{2}(\mathbb{C})$-valued distribution of $A B$ and then getting from this the (scalar-valued) distribution of $A B$ by taking the trace over $M_{2}(\mathbb{C})$. This has motivated us to look at the general problem of dealing with operator-valued free multiplicative convolutions.

The standard way to deal with free multiplicative convolutions is through Voiculescu's $S$-transform. The generalization of the $S$-transform to the operator-valued situation was found by Dykema 13. (A certain version of the $S$-transform, via a Fock space-type construction, appeared already in the work of Voiculescu [29].) Direct computations of operator-valued $S$-transforms for non-trivial elements are, however, extremely hard. Approximations can be done on domains corresponding to domains of the Cauchy-Stieltjes transform which are located far away from the real axis. For practical purposes this is a problem, since we are interested in the behavior of the Cauchy transform close to the real axis, in order to recover the distribution by an Stieltjes inversion.

In this paper we follow Biane's approach 9] to scalar-valued free multiplicative convolution via analytic subordination, which was later extended by Voiculescu 30, 31] to the operator valued case. 
Our main contribution to the theory is to find the subordination functions as iterative limits, similar to what has been done in the scalar case [2]. We rely on the twisted multiplicative property of Dykema's $S$-transform.

This will allow us to set up fixed point equations to approximate effectively the values of the Cauchy transform of $A B$ in the whole operator-valued upper half plane and in particular, close to the real axis. As inputs, we require the individual operator-valued Cauchy Transforms of $A$ and $B$ (or good approximations of these).

We want to stress that in the operator-valued context there are rarely situations where one has an analytic formula for the involved Cauchy transforms. The best one can hope for are equations which determine those transforms. In order to be applicable to concrete problems one needs of course a way to solve these equations in particular, to single out the correct solution; our operator-valued equations usually have many solutions, only one of them corresponding to the wanted Cauchy transform. Since the characterization of the Cauchy transform among all solutions is by positivity requirements this is an intrinsic analytic characterization, which indicates that those equations cannot be solved by pure formal power series expansion arguments. So what we need for a theory of operator-valued convolution which is also practically applicable is to set up our theory in such a form which can also numerically be implemented (and such that the theory provides arguments for the working of this implementation). What has become more and more apparent in the scalar-valued context, namely that the subordination formulation of free convolution seems to be the right choice, is even more prominent in the operatorvalued context. Trying to solve an operator-valued free multiplicative convolution problem directly with the help of the operator-valued $S$-transform becomes quite a challenging task very soon, whereas using the subordination formulation, as presented in this paper, allows not only a satisfying analytic description of the involved transformations, but can also be implemented numerically very easily.

In Section 2, we will present our analytic description, via subordination, of the free multiplicative operator-valued convolution. In Section 3, we will show the usefulness of our approach by implementing our method to calculate the distribution of the product of two operator-valued free random variables and by comparing this with histograms for corresponding random models. We consider there two different types of examples: (i) the product of two operator-valued semicircular elements, the individual Cauchy transforms obtained by the fixed point equations described in [14; (ii) in the context of our original problem described above, we treat the case $a b a+a^{2} b a^{2}$, where $a$ is discrete and $b$ is either discrete or a semicircular variable.

\section{MUltiplication OF OPERATOR-VALUED FREE RANDOM VARIABLES}

We will call an operator-valued non-commutative probability space a triple $(\mathcal{M}, \mathbb{E}, B)$, where $\mathcal{M}$ is a von Neumann algebra, $B \subseteq \mathcal{M}$ is a $W^{*}$-subalgebra containing the unit of $\mathcal{M}$, and $\mathbb{E}: \mathcal{M} \rightarrow B$ is a unit-preserving conditional expectation. Elements in $\mathcal{M}$ will be called operator-valued (or $B$-valued) random variables. The distribution of a random variable $x \in \mathcal{M}$ with respect to $\mathbb{E}$ is, by definition, the set of multilinear maps

$$
\mu_{x}:=\left\{m_{n}^{x}: B^{n-1} \rightarrow B: m_{n}\left(b_{1}, \ldots, b_{n-1}\right)=\mathbb{E}\left[x b_{1} x b_{2} \cdots x b_{n-1} x\right], n \in \mathbb{N}\right\} .
$$

We call $m_{n}^{x}$ the $n^{\text {th }}$ moment of $x$ (or, equivalently, of $\mu_{x}$ ). It will be convenient to interprete $m_{0}^{x}$ as the constant equal to 1 , the unit of $B$ (or, equivalently, of $\mathcal{M}$ ) 
and $m_{1}^{x}=\mathbb{E}[x]$, the expectation of $x$. We denote by $B\left\langle x_{1} \ldots, x_{n}\right\rangle$ the ${ }^{*}$-algebra generated by $B$ and the elements $x_{1}, \ldots, x_{n}$ in $\mathcal{M}$.

Definition 2.1. Two algebras $A_{1}, A_{2} \subseteq \mathcal{M}$ containing $B$ are called free with amalgamation over $B$ with respect to $\mathbb{E}$ (or just free over $B$ ) if

$$
\mathbb{E}\left[x_{1} x_{2} \cdots x_{n}\right]=0
$$

whenever $n \in \mathbb{N}, x_{j} \in A_{i_{j}}$ satisfy $\mathbb{E}\left[x_{j}\right]=0$ and $i_{j} \neq i_{j+1}, 1 \leq j \leq n-1$. Two random variables $x, y \in \mathcal{M}$ are called free over $B$ if $B\langle x\rangle$ and $B\langle y\rangle$ are free over $B$.

If $x, y \in \mathcal{M}$ are free over $B$, then $\mu_{x+y}$ and $\mu_{x y}$ depend only on $\mu_{x}$ and $\mu_{y}$. Following Voiculescu, we shall denote these dependencies by $\mu_{x} \boxplus \mu_{y}$ and $\mu_{x} \otimes \mu_{y}$, and call them the free additive, respectively free multiplicative, convolution of the distributions $\mu_{x}$ and $\mu_{y}$. It is known [20, 29] that both $\boxplus$ and $\otimes$ are associative, but $\otimes$ may fail to be commutative.

2.1. Analytic transforms. A very powerful tool for the study of operator-valued distributions is the generalized Cauchy-Stieltjes transform and its fully matricial extension [29, 30]: for a fixed $x \in \mathcal{M}$, we define $G_{x}(b)=\mathbb{E}\left[(b-x)^{-1}\right]$ for all $b \in B$ so that $b-x$ is invertible in $\mathcal{M}$. One can easily verify that $G_{x}$ is a holomorphic mapping on an open subset of $B$. Its fully matricial extension $G_{x}^{(n)}$ is defined on the set of elements $b \in M_{n}(B)$ for which $b-x \otimes 1_{n}$ is invertible in $M_{n}(\mathcal{M})$, by the relation $G_{x}^{(n)}(b)=\mathbb{E} \otimes \operatorname{Id}_{M_{n}(\mathbb{C})}\left[\left(b-x \otimes 1_{n}\right)^{-1}\right]$. It is a crucial observation of Voiculescu that the family $\left\{G_{x}^{(n)}\right\}_{n \geq 1}$ encodes the distribution $\mu_{x}$ of $x$. A succint description of how to identify the $n^{\text {th }}$ moment of $x$ when $\left\{G_{x}^{(n)}\right\}_{n \geq 1}$ is known is given in [4].

In the following we will use the notation $x>0$ for the situation where $x \geq 0$ and $x$ is invertible; note that this is equivalent to the fact that there exists a real $\varepsilon \geq 0$ such that $x \geq \varepsilon 1$. From the later it is clear that $x>0$ implies $\mathbb{E}[x]>0$ (because our conditional expectations are automatically completely positive).

From now on we shall restrict our attention to the case when $x, y$ are selfadjoint, and (for most applications) nonnegative. In this case, one of appropriate domains for $G_{x}$ - and the domain we will use most - is the operator upper halfplane $\mathbb{H}^{+}(B):=\{b \in B: \Im b>0\}$. Elements in this open set are all invertible, and $\mathbb{H}^{+}(B)$ is invariant under conjugation by invertible elements in $B$. It has been noted in 30 that $G_{x}^{(n)}$ maps $\mathbb{H}^{+}\left(M_{n}(B)\right)$ into the operatorial lower half-plane $\mathbb{H}^{-}\left(M_{n}(B)\right):=-\mathbb{H}^{+}\left(M_{n}(B)\right)$ and has "good behaviour at infinity" in the sense that $\lim _{\left\|b^{-1}\right\| \rightarrow 0} b G_{x}^{(n)}(b)=\lim _{\left\|b^{-1}\right\| \rightarrow 0} G_{x}^{(n)}(b) b=1$.

As, from an analytic function perspective, $G_{x}^{(n)}$ have essentially the same behaviour on $\mathbb{H}^{+}\left(M_{n}(B)\right)$ for any $n \in \mathbb{N}$, we shall restrict our analysis from now on to $G_{x}:=G_{x}^{(1)}$. However, all properties we deduce for this $G_{x}$, and all the related functions we shall introduce, remain true, under the appropriate formulation, for all $n \geq 1$.

We shall use the following analytic mappings, all defined on $\mathbb{H}^{+}(B)$; all transforms have a natural Schwarz-type analytic extension to the lower half-plane given by $f\left(b^{*}\right)=f(b)^{*}$; in all formulas below, $x=x^{*}$ is fixed in $\mathcal{M}$ : 
- the moment generating function:

$$
\Psi_{x}(b)=\mathbb{E}\left[(1-b x)^{-1}-1\right]=\mathbb{E}\left[\left(b^{-1}-x\right)^{-1}\right] b^{-1}-1=G_{x}\left(b^{-1}\right) b^{-1}-1
$$

- The reciprocal Cauchy transform:

$$
F_{x}(b)=\mathbb{E}\left[(b-x)^{-1}\right]^{-1}=G_{x}(b)^{-1} ;
$$

- The eta transform (Boolean cumulant series):

$\eta_{x}(b)=\Psi_{x}(b)\left(1+\Psi_{x}(b)\right)^{-1}=1-b \mathbb{E}\left[\left(b^{-1}-x\right)^{-1}\right]^{-1}=1-b F_{x}\left(b^{-1}\right) ;$

- In this paper we shall call this function "the h transform:"

$$
h_{x}(b)=b^{-1} \eta_{x}(b)=b^{-1}-\mathbb{E}\left[\left(b^{-1}-x\right)^{-1}\right]^{-1}=b^{-1}-F_{x}\left(b^{-1}\right) ;
$$

Based on the moment generating function, Dykema [13] introduced the operatorvalued version of Voiculescu's $S$-transform [26] as an analytic mapping on Banach algebras (an earlier, less easily employed version can be found in [29]). It is easy to note that $\Psi_{x}^{\prime}(b)(c)=\mathbb{E}\left[(1-b x)^{-1} c x(1-b x)^{-1}\right]$, so that $\Psi_{x}^{\prime}(0)(c)=\mathbb{E}[c x]=c \mathbb{E}[x]$. Under the assumption that $\mathbb{E}[x]$ is invertible in $B$ (and, in particular, when $x>0$ ), the linear map $\Psi_{x}^{\prime}(0)$ becomes invertible, with inverse $c \mapsto c \mathbb{E}[x]^{-1}$, and so by the usual Banach-space inverse function theorem, $\Psi_{x}$ has an inverse around zero, which we shall denote by $\Psi_{x}^{-1}$. The $S$-transform is defined as

$$
S_{x}(b)=b^{-1}(1+b) \Psi_{x}^{-1}(b), \quad\|b\| \text { small enough. }
$$

Dykema showed [13, Theorem 1.1] that, whenever $\mathbb{E}[x]$ and $\mathbb{E}[y]$ are both invertible in $B$,

$$
S_{x y}(b)=S_{y}(b) S_{x}\left(S_{y}(b)^{-1} b S_{y}(b)\right), \quad\|b\| \text { small enough. }
$$

2.2. Three ways to the subordination function. Here we shall describe three ways to finding the analytic subordination functions for free multiplicative convolution of operator-valued distributions. The analytic subordination has been proved in different contexts by Voiculescu and Biane [28, 9, 30, 31. For the case $B=\mathbb{C}$, Biane showed that there exist analytic functions $\omega_{1}, \omega_{2}: \mathbb{C} \backslash[0,+\infty) \rightarrow$ $\mathbb{C} \backslash[0,+\infty)$ which preserve half-planes and satisfy $\mathbb{E}_{\mathbb{C}\langle y\rangle}\left[(1-z x y)^{-1}\right]=(1-$ $\left.\omega_{2}(z) y\right)^{-1}, \mathbb{E}_{\mathbb{C}\langle x\rangle}\left[(1-z x y)^{-1}\right]=\left(1-\omega_{1}(z) x\right)^{-1}$. In particular, $\eta_{x}\left(\omega_{1}(z)\right)=$ $\eta_{y}\left(\omega_{2}(z)\right)=\eta_{x y}(z), z \in \mathbb{C} \backslash[0,+\infty)$. Voiculescu extended this relation, essentially in 30, and made it more precise in 31, to the case of a general $B$, under the assumption that $\mathcal{M}$ is endowed with a tracial state and $\mathbb{E}$ preserves this trace. Later, in 2], Biane's subordination functions $\omega_{1}, \omega_{2}$ were found as limits of an iteration process involving $\eta_{x}$ and $\eta_{y}$. A precise description of such an iterative process for (very general) positive operator-valued random variables, in the spirit of [14], will be our main contribution in this section.

Inspired by the shape of formula (8), we claim that for our purposes, the most appropriate writing of the operator-valued subordination phenomenon is the following:

Theorem 2.2. Let $x>0, y=y^{*} \in \mathcal{M}$ be two random variables with invertible expectations, free over $B$. There exists a Gâteaux holomorphic map $\omega_{2}:\{b \in$ $B: \Im(b x)>0\} \rightarrow \mathbb{H}^{+}(B)$, such that

(1) $\eta_{y}\left(\omega_{2}(b)\right)=\eta_{x y}(b), \Im(b x)>0$;

(2) $\omega_{2}(b)$ and $b^{-1} \omega_{2}(b)$ are analytic around zero; 
(3) For any $b \in B$ so that $\Im(b x)>0$, the map $g_{b}: \mathbb{H}^{+}(B) \rightarrow \mathbb{H}^{+}(B), g_{b}(w)=$ $b h_{x}\left(h_{y}(w) b\right)$ is well-defined, analytic and

$$
\omega_{2}(b)=\lim _{n \rightarrow \infty} g_{b}^{\circ n}(w),
$$

for any fixed $w \in \mathbb{H}^{+}(B)$.

Moreover, if one defines $\omega_{1}(b):=h_{y}\left(\omega_{2}(b)\right) b$, then

$$
\eta_{x y}(b)=\omega_{2}(b) \eta_{x}\left(\omega_{1}(b)\right) \omega_{2}^{-1}(b), \quad \Im(b x)>0 .
$$

Following the proof of this theorem, we shall mention several conditions under which some hypotheses, particularly the - rather inconvenient in practical applications - invertibility requirement, can be weakened or entirely dropped.

Proof. We shall split our proof in several remarks, formulas and lemmas. For our purposes, a slight variation of the $S$-transform will be more useful: we define the sigma transform $\Sigma_{x}(b)=b^{-1} \eta_{x}^{-1}(b)$, again on a neighbourhood of zero. Elementary arithmetic manipulations show that $\Sigma_{x}(b)=S_{x}\left(b(1-b)^{-1}\right)$ and so $\Sigma_{x y}(b)=\Sigma_{y}(b) \Sigma_{x}\left(\Sigma_{y}(b)^{-1} b \Sigma_{y}(b)\right)$. Using this relation we write on a neighbourhood of zero in $B$ :

$$
\begin{aligned}
b \eta_{x y}^{-1}(b) & =b^{2} \Sigma_{x y}(b) \\
& =b^{2} \Sigma_{y}(b) \Sigma_{x}\left(\Sigma_{y}(b)^{-1} b \Sigma_{y}(b)\right) \\
& =b^{2} \Sigma_{y}(b)\left(\Sigma_{y}(b)^{-1} b \Sigma_{y}(b)\right)^{-1} \eta_{x}^{-1}\left(\Sigma_{y}(b)^{-1} b \Sigma_{y}(b)\right) \\
& =b \Sigma_{y}(b) \eta_{x}^{-1}\left(\Sigma_{y}(b)^{-1} b \Sigma_{y}(b)\right) \\
& =\eta_{y}^{-1}(b) \eta_{x}^{-1}\left(\left(\eta_{y}^{-1}(b)\right)^{-1} b \eta_{y}^{-1}(b)\right) .
\end{aligned}
$$

Now define $\omega_{2}(b)=\eta_{y}^{-1}\left(\eta_{x y}(b)\right)$, again for $\|b\|$ sufficiently small. We substitute in the previous relation $\eta_{x y}(b)$ for $b$ to obtain

$$
\begin{aligned}
\eta_{y}\left(\omega_{2}(b)\right) b & =\eta_{x y}(b) b \\
& =\eta_{y}^{-1}\left(\eta_{x y}(b)\right) \eta_{x}^{-1}\left(\left(\eta_{y}^{-1}\left(\eta_{x y}(b)\right)\right)^{-1} \eta_{x y}(b) \eta_{y}^{-1}\left(\eta_{x y}(b)\right)\right) \\
& =\omega_{2}(b) \eta_{x}^{-1}\left(\omega_{2}(b)^{-1} \eta_{y}\left(\omega_{2}(b)\right) \omega_{2}(b)\right) .
\end{aligned}
$$

Recalling from equation (6) the definition of the $\mathrm{h}$ transform, we obtain

$$
h_{y}\left(\omega_{2}(b)\right) b=\eta_{x}^{-1}\left(h_{y}\left(\omega_{2}(b)\right) \omega_{2}(b)\right), \quad\|b\| \text { small enough. }
$$

An application of $\eta_{x}$ on both sides gives $\eta_{x}\left(h_{y}\left(\omega_{2}(b)\right) b\right)=h_{y}\left(\omega_{2}(b)\right) \omega_{2}(b)$, or

$$
\omega_{2}(b)=b h_{x}\left(h_{y}\left(\omega_{2}(b)\right) b\right)=g_{b}\left(\omega_{2}(b)\right), \quad\|b\| \text { small enough. }
$$

This shows us that $\omega_{2}$ exists on a small enough neighbourhood of the origin, and is a fixed point for the map $g_{b}$ introduced in Theorem 2.2. Moreover, this indicates that $b^{-1} \omega_{2}(b)$ is analytic around zero (a fact that follows quite easily also from the definition of $\omega_{2}$ as $\eta_{y}^{-1} \circ \eta_{x y}$ ). The most significant, however, is the fact that, under the conditions of analyticity of the two maps $\omega_{2}(b)$ and $b^{-1} \omega_{2}(b)$ around zero, equation (9) uniquely determines $\omega_{2}$, and thus a function satisfying $(9)$ must also satisfy $\eta_{y} \circ \omega_{2}=\eta_{x y}$, and vice-versa.

Now we observe that, under the additional assumption of the existence of a trace $\tau$ on $\mathcal{M}$ so that $\tau=\tau \circ \mathbb{E}$, this function coincides with a function provided by Voiculescu: in [30, Voiculescu proved that, whenever $x$ and $y$ are free over $B$, the range of the analytic map $b \mapsto y+\mathbb{E}_{B\langle y\rangle}\left[(b-x-y)^{-1}\right]^{-1}$ is included in $B$. $\left(\mathbb{E}_{B\langle y\rangle}\right.$ denotes the conditional expectation with $\tau$ onto the von Neumann 
algebra generated by $B$ and $y$.) It follows quite easily that the range of the map $b \mapsto y^{-1}-\mathbb{E}_{B\langle y\rangle}\left[\left(y^{-1}-b x\right)^{-1}\right]^{-1}$ is also included in $B$. We claim that

$$
\omega_{2}(b)=y^{-1}-\mathbb{E}_{B\langle y\rangle}\left[\left(y^{-1}-b x\right)^{-1}\right]^{-1} .
$$

Since $\omega_{2}$ up to this moment has only been defined on a neighbourhood of zero, we only need to verify the equality for $\|b\|$ small. Indeed, the above relation is equivalent to $\mathbb{E}_{B\langle y\rangle}\left[(1-b x y)^{-1}\right]^{-1}=1-\omega_{2}(b) y$, by the bimodule property of $\mathbb{E}_{B\langle y\rangle}$. Inverting both sides of the equality and applying the conditional expectation $\mathbb{E}$ gives $\mathbb{E}\left[(1-b x y)^{-1}\right]=\mathbb{E}\left[\left(1-\omega_{2}(b) y\right)^{-1}\right]$. We invert, take a $b$ as a factor and subtract 1 in order to get $\eta_{y}\left(\omega_{2}(b)\right)=\eta_{x y}(b)$. As it was noted by Voiculescu 30, and we shall argue below, $y^{-1}-b x$ is invertible whenever $\Im(b x)>0$. Since the set $\{b \in B: \Im(b x)>0\} \cap\{b \in B:\|b\|<\varepsilon\}$ is open for any $\varepsilon>0$, and $\lim _{\|b\| \rightarrow 0}\left(y^{-1}-\mathbb{E}_{B\langle y\rangle}\left[\left(y^{-1}-b x\right)^{-1}\right]^{-1}\right)=0$, it follows, by analytic continuation, that there exists an $\varepsilon>0$ so that

$$
\begin{gathered}
\omega_{2}:\{b \in B: \Im(b x)>0\} \cup\{b \in B:\|b\|<\varepsilon\} \rightarrow B \\
\omega_{2}(b)=y^{-1}-\mathbb{E}_{B\langle y\rangle}\left[\left(y^{-1}-b x\right)^{-1}\right]^{-1} .
\end{gathered}
$$

Until now we have argued that an analytic map $\omega_{2}$ satisfying parts (1) and (2) of our theorem exists and is unique (i) on a neighbourhood of zero for any $B$ valued non-commutative probability space $(\mathcal{M}, \mathbb{E}, B)$, and (ii) that this $\omega_{2}$ extends analytically to $\{b \in B: \Im(b x)>0\}$ when $\mathcal{M}$ is endowed with a tracial state $\tau$ so that $\tau \circ \mathbb{E}$ remains a trace. We go now to the third way of identifying $\omega_{2}$, namely as a fixed point of an analytic mapping, method which will allow us to extend $\omega_{2}$ to a set of the form $\{b \in B: \Im(b x)>0\} \cup\{b \in B:\|b\|<\varepsilon\}$ for any type of non-commutative probability space $(\mathcal{M}, \mathbb{E}, B)$.

Lemma 2.3. Assume that $x \geq 0$ is invertible in $\mathcal{M}$ and $b \in\{b \in B: \Im(b x)>0\}$. If $c \in B$ is so that $\Im c \geq 0$, then $\Im h_{b x}(c) \geq \mathbb{E}\left[(\Im(b x))^{-1}\right]^{-1}>0$.

Proof. For simplicity, let us replace $c \in \mathbb{H}^{+}(B)$ by $-c^{-1} \in \mathbb{H}^{+}(B)$, so that

$$
h_{b x}\left(-c^{-1}\right)=\mathbb{E}\left[(c+b x)^{-1}\right]^{-1}-c .
$$

We shall split our problem in two and use the same method as in [4]: assume that $\varphi$ is an arbitrary positive linear functional on $B$ so that $\varphi(1)=1$. We define

$$
f_{\varphi}: \mathbb{C}^{+} \rightarrow \mathbb{C}^{+}, \quad f_{\varphi}(z)=\varphi\left(\mathbb{E}\left[(\Re(c+b x)+z \Im(c+b x))^{-1}\right]^{-1}\right) .
$$

As $\Im c \geq 0$, and $\Im(b x), \Im z$ are all strictly positive, it follows that $\Im(\Re(c+b x)+z \Im(c+$ $b x))^{-1}<0$, and, since $\mathbb{E}$ is positive and faithful, $\Im \mathbb{E}\left[(\Re(c+b x)+z \Im(c+b x))^{-1}\right]<$ 0. Thus, $\Im\left(\mathbb{E}\left[(\Re(c+b x)+z \Im(c+b x))^{-1}\right]^{-1}\right)>0$, and, in particular, invertible. Since $\varphi \geq 0, \varphi(1)=1$, the Cauchy-Schwarz inequality tells us that $\Im f_{\varphi}(z)>0$ whenever $\Im z>0$. We take

$$
\begin{aligned}
\lim _{z \rightarrow \infty} \frac{f_{\varphi}(z)}{z} & =\varphi\left(\lim _{z \rightarrow \infty} \mathbb{E}\left[\left(\frac{\Re(c+b x)}{z}+\Im(c+b x)\right)^{-1}\right]^{-1}\right) \\
& =\varphi\left(\mathbb{E}\left[(\Im(c+b x))^{-1}\right]^{-1}\right) .
\end{aligned}
$$


The Nevanlinna representation [1, Chapter III] allows us to write the inequality $\Im f_{\varphi}(z) \geq \varphi\left(\mathbb{E}\left[(\Im(c+b x))^{-1}\right]^{-1}\right) \Im z$ for all $z \in \mathbb{C}^{+}$. Equality holds at one point of $\mathbb{C}^{+}$if and only if $f_{\varphi}(z)=\varphi\left(\mathbb{E}\left[(\Im(c+b x))^{-1}\right]^{-1}\right) z+$ real constant. Taking $z=i$ in the above we get

$$
\Im \varphi\left(\mathbb{E}\left[(c+b x)^{-1}\right]^{-1}\right) \geq \varphi\left(\mathbb{E}\left[(\Im(c+b x))^{-1}\right]^{-1}\right) .
$$

Since this inequality holds for all states $\varphi$ on $B$, we conclude that

$$
\Im \mathbb{E}\left[(c+b x)^{-1}\right]^{-1} \geq \mathbb{E}\left[(\Im(c+b x))^{-1}\right]^{-1} .
$$

We shall now prove that $\mathbb{E}\left[(\Im(c+b x))^{-1}\right]^{-1}>\Im c$ :

$$
\begin{aligned}
\mathbb{E} & {\left[(\Im(c+b x))^{-1}\right]^{-1}>\Im c \Longleftrightarrow \mathbb{E}\left[(\Im c+\Im(b x))^{-1}\right]<(\Im c)^{-1} } \\
& \Longleftrightarrow \mathbb{E}\left[\sqrt{\Im c}(\Im c+\Im(b x))^{-1} \sqrt{\Im c}\right]<1 \\
& \Longleftrightarrow \mathbb{E}\left[\left(1+(\sqrt{\Im c})^{-1} \Im(b x)(\sqrt{\Im c})^{-1}\right)^{-1}\right]<1 \\
& \Longleftarrow\left(1+(\sqrt{\Im c})^{-1} \Im(b x)(\sqrt{\Im c})^{-1}\right)^{-1}<1 .
\end{aligned}
$$

The last inequality is trivially true by functional calculus and the invertibility of $\Im(b x)$. Putting the inequalities together gives

$$
\varphi\left(\Im \mathbb{E}\left[(c+b x)^{-1}\right]^{-1}\right)=\Im \varphi\left(\mathbb{E}\left[(c+b x)^{-1}\right]^{-1}\right)>\varphi(\Im c) .
$$

Since this is true for all positive $\varphi$, we get that $h_{b x}$ maps $\mathbb{H}^{+}(B)$ into itself.

Next, we make use again of the same trick: we define

$$
f_{\varphi}: \mathbb{C}^{+} \rightarrow \mathbb{C}^{+}, \quad f_{\varphi}(z)=\varphi\left(\mathbb{E}\left[(c+\Re(b x)+z \Im(b x))^{-1}\right]^{-1}-c\right) .
$$

As before, whenever $\Im c \geq 0$ (the case $c=c^{*}$, for example, is not excluded here), $\Im \mathbb{E}\left[(c+\Re(b x)+z \Im(b x))^{-1}\right]^{-1}-\Im c>0$ for any $z \in \mathbb{C}^{+}$. We take again limit as $z \rightarrow \infty$ of $f_{\varphi}(z) / z$ to obtain $\varphi\left(\mathbb{E}\left[(\Im(b x))^{-1}\right]^{-1}\right)$. The same argument used above implies that $\Im f_{\varphi}(z) \geq \varphi\left(\mathbb{E}\left[(\Im(b x))^{-1}\right]^{-1}\right) \Im z$, and so, for $z=i$ we obtain $\Im \varphi\left(\mathbb{E}\left[(c+\Re(b x)+i \Im(b x))^{-1}\right]^{-1}-c\right) \geq \varphi\left(\mathbb{E}\left[(\Im(b x))^{-1}\right]^{-1}\right)$, for all $\varphi \geq 0, \varphi(1)=1$. This implies $\Im h_{b x}(c) \geq \mathbb{E}\left[(\Im(b x))^{-1}\right]^{-1}$, as claimed.

By the definition of the $\mathrm{h}$ transform (6), we have $b h_{x}(c b)=h_{b x}(c)$. The previous lemma allows us to write

$$
\Im h_{y}(w) \geq 0 \Longrightarrow \Im h_{b x}\left(h_{y}(w)\right) \geq \mathbb{E}\left[(\Im(b x))^{-1}\right]^{-1}>0,
$$

i.e. $\Im g_{b}(w) \geq \mathbb{E}\left[(\Im(b x))^{-1}\right]^{-1}>0$ for any $w \in \mathbb{H}^{+}(B)$, and $b$ with $\Im(b x)>0$. Thus, we have shown that $g_{b}$ lands strictly into $\mathbb{H}^{+}(B)$ whenever $\Im(b x)>0$ and $\Im h_{y}(w) \geq 0$.

Remark 2.4. It has been shown in [4] that $\Im \mathbb{E}\left[(w-y)^{-1}\right]^{-1} \geq \Im w$ for any $w \in$ $\mathbb{H}^{+}(B)$ and $y=y^{*} \in \mathcal{M}$. By noting that $-w^{-1} \in \mathbb{H}^{+}(B)$ if and only if $w \in \mathbb{H}^{+}(B)$, we obtain that $\Im h_{y}(w) \geq 0$ and $\Im h_{y}\left(-w^{-1}\right) \geq 0$ for any $w \in \mathbb{H}^{+}(B)$. 
We can now improve on our previous statement: for $x>0, y=y^{*}$, and $b \in B$ so that $\Im(b x)>0$,

$$
\Im w>0 \Longrightarrow \Im h_{y}(w) \geq 0 \Longrightarrow \Im h_{b x}\left(h_{y}(w)\right) \geq \mathbb{E}\left[(\Im(b x))^{-1}\right]^{-1}>0,
$$

i.e. $\Im g_{b}(w) \geq \mathbb{E}\left[(\Im(b x))^{-1}\right]^{-1}>0$ for any $w \in \mathbb{H}^{+}(B)$, and $b$ with $\Im(b x)>0$.

Remark 2.5. The functions $h$ associated to selfadjoints $y \in \mathcal{M}$ have convergent power series expansions around the origin. Indeed,

$$
\begin{aligned}
\mathbb{E} & {[1-w y]-\mathbb{E}\left[(1-w y)^{-1}\right]^{-1} } \\
& =\mathbb{E}[1-w y]\left(\mathbb{E}\left[(1-w y)^{-1}\right]-E[1-w y]^{-1}\right) \mathbb{E}\left[(1-w y)^{-1}\right]^{-1} \\
& =\mathbb{E}[1-w y] w\left(\sum_{n=1}^{\infty} \mathbb{E}\left[y(w y)^{n}\right]-\mathbb{E}[y] \mathbb{E}[w y]^{n}\right) \mathbb{E}\left[(1-w y)^{-1}\right]^{-1} .
\end{aligned}
$$

Thus,

$$
\begin{aligned}
h_{y}(w)= & w^{-1}\left(1-\mathbb{E}\left[(1-w y)^{-1}\right]^{-1}\right) \\
= & w^{-1}\left[\mathbb{E}[1-w y] w\left(\sum_{n=1}^{\infty} \mathbb{E}\left[y(w y)^{n}\right]-\mathbb{E}[y] \mathbb{E}[w y]^{n}\right) \mathbb{E}\left[(1-w y)^{-1}\right]^{-1}\right] \\
& +w^{-1} \mathbb{E}[w y] \\
= & (1-\mathbb{E}[y w])\left[\left(\sum_{n=1}^{\infty} \mathbb{E}\left[y(w y)^{n}\right]-\mathbb{E}[y] \mathbb{E}[w y]^{n}\right) \mathbb{E}\left[(1-w y)^{-1}\right]^{-1}\right]+\mathbb{E}[y]
\end{aligned}
$$

which gives the power series espansion of $h_{y}$ around zero and shows that $h_{y}(0)=$ $\mathbb{E}[y]$.

We note that, as shown in the above Remark, there exists a $\varepsilon>0$ so that $h_{x}, h_{y}$ are defined on $\{w \in B:\|w\|<\varepsilon\}$ and

$$
\max \left\{\left\|h_{x}(w)\right\|,\left\|h_{y}(w)\right\|: w \in B,\|w\|<\varepsilon\right\}<2(\|x\|+\|y\|) .
$$

Choosing $b \in B$ with $\|b\|<(\varepsilon / 41) \cdot(\|x\|+\|y\|)^{-1}$ guarantees that $\left\|h_{y}(w) b\right\|<\varepsilon / 10$ and $\left\|b h_{x}\left(h_{y}(w) b\right)\right\|<\varepsilon / 2$, so $g_{b}$ maps $\{w \in B:\|w\|<\varepsilon\}$ into $\{w \in B:\|w\|<$ $\varepsilon / 2\}$. Thus, by the Earle-Hamilton Theorem [12, Theorem 11.1], $g_{b}$ has a unique attracting fixed point in $\{w \in B:\|w\|<\varepsilon\}$ which we shall denote by $\omega_{2}(b)$, and

$$
\lim _{n \rightarrow \infty} g_{b}^{\circ n}(w):=\omega_{2}(b)
$$

exists for all $w \in\{w \in B:\|w\|<\varepsilon\},\|b\|<(\varepsilon / 41) \cdot(\|x\|+\|y\|)^{-1}$. The correspondence $b \mapsto \omega_{2}(b)$ is clearly analytic, being a uniform limit of analytic maps. Moreover, on a small enough neighbourhood of zero, $\eta_{y}\left(\omega_{2}(b)\right)=\eta_{x y}(b)$ by the uniqueness of the fixed point guaranteed by the Earle-Hamilton Theorem.

For our fixed $x, y \in \mathcal{M}$ given in the statement of our theorem, let us fix an $\varepsilon>0$ as above. Consider the set

$$
\left\{b \in B:\|b\|<\frac{\varepsilon}{41(\|x\|+\|y\|)}\right\} \cup\left\{b \in B: \Im(b x)>\frac{\varepsilon}{99\left\|x^{-1}\right\|(\|x\|+\|y\|)}\right\} .
$$

This set is clearly open and connected (we can find the element $b=i \frac{\varepsilon}{82\left\|x^{-1}\right\|(\|x\|+\|y\|)}$ in both open sets whose union we considered). The above indicates that 
(1) $g:\left\{b \in B:\|b\|<\frac{\varepsilon}{41(\|x\|+\|y\|)}\right\} \times\{w \in B:\|w\|<\varepsilon\} \rightarrow\left\{w \in B:\|w\|<\frac{\varepsilon}{2}\right\}$ given by $g_{b}(w)=h_{b x}\left(h_{y}(w)\right)$ is analytic;

(2) $g:\left\{b \in B: \Im(b x)>\frac{\varepsilon}{99\left\|x^{-1}\right\|(\|x\|+\|y\|)}\right\} \times\left\{w \in B: \Im w>\frac{\varepsilon}{200\left\|x^{-1}\right\|(\|x\|+\|y\|)}\right\}$ $\rightarrow\left\{w \in B: \Im w>\mathbb{E}\left[(\Im(b x))^{-1}\right]^{-1}\right\}$ given by $g_{b}(w)=h_{b x}\left(h_{y}(w)\right)$ is analytic.

The union of the two domains of $g$ mentioned above is again connected, as we immediately note by identifying the point $(b, w)=\left(\frac{i \varepsilon}{82\left\|x^{-1}\right\|(\|x\|+\|y\|)}, \frac{i \varepsilon}{2}\right)$ in both sets. Since the following chain of implications holds,

$$
\Im(b x)>\kappa \Longrightarrow(\Im(b x))^{-1}<\frac{1}{\kappa} \Longrightarrow \mathbb{E}\left[(\Im(b x))^{-1}\right]<\frac{1}{\kappa} \Longrightarrow \mathbb{E}\left[(\Im(b x))^{-1}\right]^{-1}>\kappa,
$$

the strict inclusion

$$
\left\{w \in B: \Im w>\mathbb{E}\left[(\Im(b x))^{-1}\right]^{-1}\right\} \subset\left\{w \in B: \Im w>\frac{\varepsilon}{200\left\|x^{-1}\right\|(\|x\|+\|y\|)}\right\}
$$

holds whenever $\Im(b x)>\frac{\varepsilon}{99\left\|x^{-1}\right\|(\|x\|+\|y\|)}$.

Next, for any positive linear functional $\varphi$ on $B$ and $b \in B$ so that $\Im(b x)>$ $\frac{\varepsilon}{99\left\|x^{-1}\right\|(\|x\|+\|y\|)}$, we shall show that $\left\{\varphi\left(g_{b}^{\circ n}(w)\right)\right\}_{n \in \mathbb{N}}$ is bounded. The argument uses the fact that the set $\{b \in B: \Im(b x)>0\}$ is convex. we choose $b_{1} \in B$, $\Im\left(b_{1} x\right)>\frac{\varepsilon}{99\left\|x^{-1}\right\|(\|x\|+\|y\|)},\left\|b_{1}\right\|<\frac{\varepsilon}{41(\|x\|+\|y\|)}$ and consider $t \mapsto \varphi\left(g_{t b+(1-t) b_{1}}^{\circ n}(w)\right)$. The map $[0,1] \ni t \mapsto g_{t b+(1-t) b_{1}}^{\circ n}(w)$ lands, for a fixed $w \in \mathbb{H}^{+}(B)$, entirely in $\mathbb{H}^{+}(B)+i \frac{\varepsilon}{99\left\|x^{-1}\right\|(\|x\|+\|y\|)}$, independently of $n \in \mathbb{N}$. Thus, there exists a small enough simply connected complex neighbourhood $V$ of $[0,1]$, which does not depend on $n$ so that $V \ni t \mapsto g_{t b+(1-t) b_{1}}^{\circ n}(w)$ still lands in $\mathbb{H}^{+}(B)$ for all $n \in \mathbb{N}$. We obtain that all maps in the family

$$
\left\{V \ni t \mapsto \varphi\left(g_{t b+(1-t) b_{1}}^{\circ n}(w)\right)\right\}_{n \in \mathbb{N}}
$$

take values in $\overline{\mathbb{C}^{+}}$. This means that the family is normal (as a family of functions between complex domains). On the other hand, for $|t|$ very small, we know that

$$
\lim _{n \rightarrow \infty} g_{t b+(1-t) b_{1}}^{\circ n}(w)=\omega_{2}\left(t b+(1-t) b_{1}\right) \in B
$$

which, in particular, means that $\lim _{n \rightarrow \infty} \varphi\left(g_{t b+(1-t) b_{1}}^{\circ n}(w)\right)=\varphi\left(\omega_{2}\left(t b+(1-t) b_{1}\right)\right)$ exists and is finite. This, together with the above argued normality, implies that $\lim _{n \rightarrow \infty} \varphi\left(g_{t b+(1-t) b_{1}}^{\circ n}(w)\right)$ exists as a holomorphic function from the given neighbourhood $V$ of $[0,1]$ to $\mathbb{C}^{+}$. Now any linear functional on $B$ has a unique Jordan decomposition as a linear combination of four positive linear functionals. Thus, for any $\varphi$ in the dual of $B$, the family $\left\{\left|\varphi\left(g_{t b+(1-t) b_{1}}^{\circ n}(w)\right)\right|: t \in V, n \in \mathbb{N}\right\}$ is bounded. The uniform boundedness principle (see, for example, [23, Lemma 1]) guarantees that $\left\{\left\|g_{t b+(1-t) b_{1}}^{\circ n}(w)\right\|: t \in V, n \in \mathbb{N}\right\}$ is bounded. The fact that $B$ is a von Neumann algebra implies that $\left\{g_{b}^{\circ n}(w)\right\}_{n \in \mathbb{N}}$ must have a w-convergent subsequence. Since $\Im g_{b}^{\circ n}(w)>\mathbb{E}\left[(\Im(b x))^{-1}\right]^{-1}$, it is clear that any such limit point must belong to $\mathbb{H}^{+}(B)$. However, as noted before, $\lim _{n \rightarrow \infty} \varphi\left(g_{t b+(1-t) b_{1}}^{\circ n}(w)\right)$ exists for all $t \in V$, $\varphi \in B^{*}$. Thus, there can only be one limit point, i.e. $\lim _{n \rightarrow \infty} g_{b}^{\text {on }}(w)=\omega_{2}(b)$ must exist for all $b$ with $\Im(b x)>0$.

Note that in fact we have shown more: the limit function $\omega_{2}(b):=\lim _{n \rightarrow \infty} g_{b}^{\circ n}(w)$ is Gâteaux holomorphic [11, Definition 2.1] on all of the set $\{b \in B: \Im(b x)>0\}$. It 
is known to be holomorphic close to the origin of $B$, where $\eta_{y}\left(\omega_{2}(b)\right)=\eta_{x y}(b)$. Using the same convexity trick as in the previous paragraph and the identity principle for the usual (scalar) analytic functions, we conclude that

$$
\eta_{y}\left(\omega_{2}(b)\right)=\eta_{x y}(b) \text { and } g_{b}\left(\omega_{2}(b)\right)=\omega_{2}(b), \quad \Im(b x)>0 .
$$

Thus, we have proved parts (1)-(3) of our theorem. The property of $\omega_{1}$ is trivial.

The above theorem, as stated, has the inconvenience that it does not cover the posssible case of non-invertible positive $x$ and the case of non-invertible $\mathbb{E}[y]$. However, when $B$ is finite dimensional (a matrix algebra), we can use normality of some families of analytic maps from $\mathbb{C}^{N}$ to itself to considerably improve our result. We shall use the same notations as in Theorem 2.2

Proposition 2.6. Let $B$ be finite-dimensional. For any $x \geq 0, y=y^{*}$ free over $B$, there exists a domain $\mathcal{D} \subset B$ containing $\mathbb{C}^{+} .1$ and an analytic map $\omega_{2}: \mathcal{D} \rightarrow \mathbb{H}^{+}(B)$ so that

$$
\eta_{y}\left(\omega_{2}(b)\right)=\eta_{x y}(b) \text { and } g_{b}\left(\omega_{2}(b)\right)=\omega_{2}(b), \quad b \in \mathcal{D} .
$$

Moreover, $\omega_{2}(b)=\lim _{n \rightarrow \infty} g_{b}^{\text {on }}(w)$ for any $w \in \mathbb{H}^{+}(B), b \in \mathcal{D}$.

Proof. We shall take as $\mathcal{D}=\operatorname{int}\{b \in B: \Im(b x) \geq 0, b$ invertible in $B\}$. By Remark 2.4. $g_{b}: \mathbb{H}^{+}(B) \rightarrow \mathbb{H}^{+}(B)$ is well-defined and analytic for any $b$ with $\Im(b x) \geq 0$. The existence of an attracting fixed point for $g_{b}$ when $b$ is very close to the origin follows by exactly the same argument as in the proof of Theorem 2.2. Since the family $\{\mathcal{D} \ni$ $\left.b \mapsto g_{b}^{\circ n}(w)\right\}_{n \in \mathbb{N}}$ is normal, we conclude as before that $\omega_{2}(b):=\lim _{n \rightarrow \infty} g_{b}^{\circ n}(w)$ exists and is analytic. (Here the fact that $\operatorname{dim}(B)<\infty$ is essential!) By the identity principle, it follows that $\omega_{2}(b)=g_{b}\left(\omega_{2}(b)\right)$ for all $b \in \mathcal{D}$, as the relation is known to hold for $b$ of small norm.

Up to this point, we have shown the existence of an $\omega_{2}$ defined on the open set $\mathcal{D} \supset \mathbb{C}^{+} \cdot 1$ which satisfies $g_{b}\left(\omega_{2}(b)\right)=\omega_{2}(b)$, independently from the invertibility of either $x$ or $\mathbb{E}[y]$. Since $\operatorname{dim}(B)<\infty$, the spectrum of $\mathbb{E}[y]$ is a finite set in $\mathbb{R}$, so it follows that non-invertibility of $\mathbb{E}[y]$ is equivalent to $\mathbb{E}[y] \in B$ having zero as eigenvalue. For any $\varepsilon>0, \mathbb{E}[y+\varepsilon \cdot 1]=\mathbb{E}[y]+\varepsilon \cdot 1$ is then invertible in $B$, and so is $x+\varepsilon \cdot 1$ in $\mathcal{M}$. If $g_{b, \varepsilon}(w)=h_{b(x+\varepsilon)}\left(h_{y+\varepsilon}(w)\right), w \in \mathbb{H}^{+}(B), \Im(b(x+\varepsilon))>0$, it follows that $g_{b, \varepsilon} \rightarrow g_{b}$ uniformly on compact subsets of $\mathbb{H}^{+}(B)$ as $\varepsilon \rightarrow 0$. Since for $b$ of small norm, $\omega_{2}(b)$ is an attracting fixed point for $g_{b}$, it follows that the small attracting fixed points of $g_{b, \varepsilon}$, which we shall call $\omega_{2}^{\varepsilon}(b)$, converge to $\omega_{2}(b)$. Normality allows us to conclude that $\omega_{2}^{\varepsilon} \rightarrow \omega_{2}$.

On the other hand, as seen in Theorem 2.2 .

$$
\eta_{y+\varepsilon} \circ \omega_{2}^{\varepsilon}=\eta_{(x+\varepsilon)(y+\varepsilon)}, \quad \varepsilon>0 .
$$

Since $\eta_{y+\varepsilon} \rightarrow \eta_{y}$ and $\eta_{(x+\varepsilon)(y+\varepsilon)} \rightarrow \eta_{x y}$, we conclude that

$$
\eta_{y} \circ \omega_{2}=\eta_{x y} \quad \text { on } \mathcal{D} \text {. }
$$

\section{Numerical Implementation}

We now consider some of the practical implications of Theorem 2.2 in the computation of the free multiplicative convolution of operator-valued random variables - in particular, those which are represented by $n \times n$ matrices. The general frame for those examples will be the following. Our problems will be given (directly or after 
some manipulations) by two matrices $x=\left(x_{i j}\right)$ and $y=\left(y_{i j}\right)$ where the entries of those matrices are living in some non-commutative probability space $(\mathcal{A}, \tau)$, and where, with respect to $\tau$, the entries of $x$ are free from the entries of $y$.

Let us set $\mathcal{M}:=M_{n}(\mathcal{A})=M_{n}(\mathbb{C}) \otimes \mathcal{A}$ and consider the trace $\varphi:=\operatorname{tr} \otimes \tau: \mathcal{M} \rightarrow \mathbb{C}$ and the conditional expectation $\mathbb{E}:=\mathrm{id} \otimes \tau: \mathcal{M} \rightarrow M_{n}(\mathbb{C})$.

Thus $x$ and $y$ are elements in the non-commutative probability space $(\mathcal{M}, \varphi)$ and we are actually interested in the scalar-valued distribution of $z:=x y$ (or some variant thereof) with respect to $\varphi=\operatorname{tr} \otimes \tau$. However, the freeness between the entries of $x$ and the entries of $y$ with respect to $\tau$ does in general not ensure freeness between $x$ and $y$ with respect to $\operatorname{tr} \otimes \tau$. What it implies is operator-valued freeness between $x$ and $y$ in the non-commutative operator-valued probability space $\left(\mathcal{M}, \mathbb{E}, M_{n}(\mathbb{C})\right)$. Thus we can first calculate the operator-valued distribution of $z$ with respect to $\mathbb{E}=\mathrm{id} \otimes \tau$ by using our free convolution results and then get the scalar-valued distribution of $z$ with respect to $\varphi=\operatorname{tr} \otimes \tau$ by applying the trace. More specifically, if $G_{z}$ is the $M_{n}(\mathbb{C})$-valued Cauchy transform of $z$, then $\operatorname{tr}\left(G_{z}\right)$ is the scalar-valued Cauchy-transform of $z$. Thus the spectral distribution of $z$ with respect to $\operatorname{tr} \otimes \tau$ is given, by virtue of the Cauchy-Stieltjes inversion formula, by:

$$
d \mu(t)=\lim _{\epsilon \rightarrow 0^{+}} \frac{-1}{\pi} \Im\left(\operatorname{tr}\left(G_{z}\left((t+i \epsilon) I_{n}\right)\right)\right), t \in \mathbb{R}
$$

where $I_{n}$ is the $n \times n$ identity matrix and $\operatorname{tr}:=\frac{1}{n} \operatorname{Tr}$ is the normalized trace on $n \times n$ matrices. So our objective requires that we compute the Cauchy transform of $x y$ at points of the form $z I_{n}$, where $z \in \mathbb{C}$.

Note now that by (4) and (6), we have:

$$
G_{x y}\left(z I_{n}\right)=\left(z I_{n}-h_{x y}\left(z^{-1} I_{n}\right)\right)^{-1}
$$

so computing $G_{x y}$ is equivalent to computing $h_{x y}$. Moreover, by Theorem 2.2 and (5) we see that

$$
z h_{x y}\left(z I_{n}\right)=\omega_{2}\left(z I_{n}\right) h_{y}\left(\omega_{2}\left(z I_{n}\right)\right)
$$

Theorem 2.2 expresses the function $\omega_{2}$ as the limit of iteratively composing the function $g_{b}$ with itself. (Since in our case $B=M_{n}(\mathbb{C})$ is finite-dimenisonal, we are actually in the realm of Prop. 2.6, and thus do not have to bother about the invertibility assumptions.) It is now clear that given the operator-valued $h$ transform of $x$ and $y$, we can numerically compute the $h_{x y}$, and thus the spectral distribution of the product $x y$. We consider several concrete examples below, in Sections 3.1 and 3.2 .

One obstacle to applying this technique to general problems is the difficulty in analytically computing the $h$ transform of many elements. It is easy to find the exact expression for the $h$ transform of discrete distributions (see Section 3.2), and numerical methods exist to compute the $h$ transform in other cases (see Section 3.1 for example).

3.1. The Product of Two Free Operator-Valued Semicirculars. Let $s_{1}, s_{2}$, $s_{3}$, and $s_{4}$ be free, semi-circular random variables, in some scalar-valued noncommutative probability space $(\mathcal{A}, \tau)$. Consider the matrices $S_{1}$ and $S_{2}$ defined 
by:

$$
S_{1}=\left(\begin{array}{ll}
s_{1} & s_{1} \\
s_{1} & s_{2}
\end{array}\right), S_{2}=\left(\begin{array}{cc}
s_{3}+s_{4} & 2 s_{4} \\
2 s_{4} & s_{3}-3 s_{4}
\end{array}\right)
$$

Matrices $S_{1}$ and $S_{2}$ represent limits of random matrices, where $s_{1}, \ldots, s_{4}$ are replaced by independent Gaussian random matrices.

As before, we set $\mathcal{M}:=M_{2}(\mathcal{A})=M_{2}(\mathbb{C}) \otimes \mathcal{A}$ and consider the $\operatorname{trace} \varphi:=\operatorname{tr} \otimes \tau:$ $\mathcal{M} \rightarrow \mathbb{C}$ and the conditional expectation $\mathbb{E}:=\tau \otimes$ id $: \mathcal{M} \rightarrow M_{2}(\mathbb{C})$.

We wish to compute the spectral distribution of $\left(S_{2}+c I_{2}\right) S_{1}$ in the scalarvalued probability space $(\mathcal{M}, \varphi)$, where $c$ is some constant chosen large enough to make $S_{2}+c I_{2}$ positive. Since $S_{1}$ and $S_{2}$ are not free in $(\mathcal{M}, \varphi)$ we cannot invoke usual free probability theory to achieve our goal. However, $S_{1}$ and $S_{2}$ are free operator-valued semicircular elements in the operator-valued probability space $\left(\mathcal{M}, \mathbb{E}, M_{2}(\mathbb{C})\right)$. Thus we can do the calculations on the operator-valued level and in the end go down to the scalar-valued level by taking the trace.

The first task is to compute the operator-valued $h$ transforms, $h_{S_{1}}$ and $h_{S_{2}}$. In this case, an analytic equation for the $h$ transforms is difficult to achieve. However, we can compute these $h$ transforms numerically using the method described in 14. In brief, this involves expressing the Cauchy transform of the operator-valued semicircular in terms of the fixed point of a contraction mapping. Specifically, if we define

$$
W(b)=\lim _{n \rightarrow \infty} \mathcal{F}_{b}^{\circ n}\left(W_{0}\right)
$$

where $\mathcal{F}_{b}(W)=(-i b+\mathbb{E}[S b S])^{-1}$, then $G_{S}(b)=-i W(b)$. Note that we require the initial state $W_{0}$ to satisfy $\Im\left(W_{0}\right)>0$; convergence of the above iteration scheme is ensured by arguments from [14. In our case, with $b=\left(b_{i j}\right)_{i, j=1}^{2}$, we have

and

$$
\mathbb{E}\left[S_{1} b S_{1}\right]=\left(\begin{array}{cc}
b_{11}+b_{12}+b_{21}+b_{22} & b_{11}+b_{21} \\
b_{11}+b_{12} & b_{11}+b_{22}
\end{array}\right)
$$

$$
\mathbb{E}\left[S_{2} b S_{2}\right]=\left(\begin{array}{lc}
2 b_{11}+2 b_{21}+2 b_{12}+4 b_{22} & 2 b_{11}+-2 b_{12}+4 b_{21}-6 b_{22} \\
2 b_{11}+4 b_{12}-2 b_{21}-6 b_{22} & 4 b_{11}-6 b_{12}-6 b_{21}+10 b_{22}
\end{array}\right)
$$

We compare the spectral distribution of $S_{1}$ and $S_{2}$ computed using this method and the Cauchy-Stieltjes inversion formula to random matrix simulations in Fig. 1 .

Finally, using the numerically computed $h$ transforms of $S_{1}$ and $S_{2}+c I_{2}$ we used the iterative method discussed here to compute the $h$ transform of their product. In Figure 1. we compare the distribution computed using our method to random matrix simulations of the ground truth spectral distribution of $\sqrt{S_{2}+c I_{2}} S_{1} \sqrt{S_{2}+c I_{2}}$.

For the sake of variety, we consider another operator-valued semi-circular example. Let now $\left\{s_{i}\right\}_{i=1}^{6}$ be free semi-circular elements, and let:

$$
S_{1}^{\prime}=\left(\begin{array}{ccc}
-10 s_{1} & 2 s_{2} & 30 s_{3} \\
2 s_{2} & -4 s_{3} & 5 s_{1} \\
30 s_{3} & 5 s_{1} & 16 s_{1}
\end{array}\right) \text { and } S_{2}^{\prime}=\left(\begin{array}{ccc}
-2 s_{4}+3 s_{6} & 3 s_{5}+30 s_{6} & s_{6} \\
3 s_{5}+30 s_{6} & s_{4}+s_{5}+s_{6} & s_{4} \\
s_{6} & s_{4} & 40 s_{4}
\end{array}\right)
$$

We follow the same pattern as previously: applying the numerical method proposed in [14] to compute the individual $h$ transforms of $S_{1}^{\prime}$ and $S_{2}^{\prime}$ (see Figure 

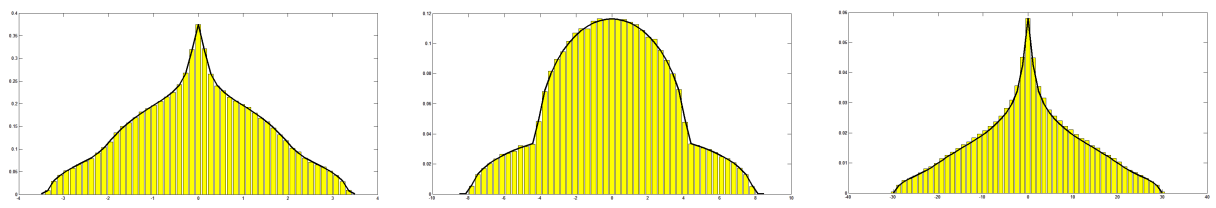

Figure 1. Spectral distribution of $S_{1}$ (left), $S_{2}$ (middle), and $\left(S_{2}+8.5 I_{2}\right) S_{1}$ (right) - random matrix simulations (histogram) compared with numerically calculated density, using fixed point method of 14] for $S_{1}$ and $S_{2}$ and using our method for $\left(S_{2}+\right.$ $\left.8.5 I_{2}\right) S_{1}$.

2), and then using our iterative method to compute the spectral distributions of $\left(S_{2}^{\prime}+85 I_{3}\right)\left(S_{1}^{\prime}+40 I_{3}\right)$ and $\left(S_{2}^{\prime}+85 I_{3}\right)\left(S_{1}^{\prime}+75 I_{3}\right)$ (see Figure 3 ).
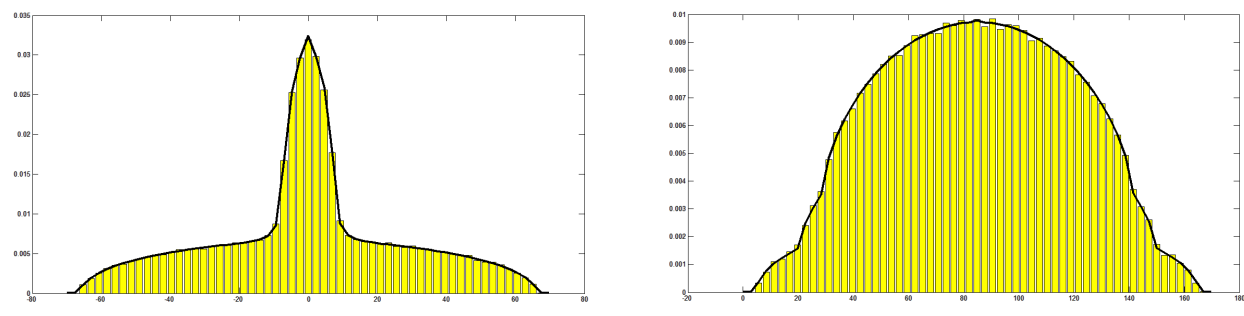

FiguRE 2. Spectral distribution of $S_{1}^{\prime}$ (left) and $S_{2}^{\prime}$ (right) - random matrix simulations (histogram) compared with numerically calculated density using fixed point method of [14].
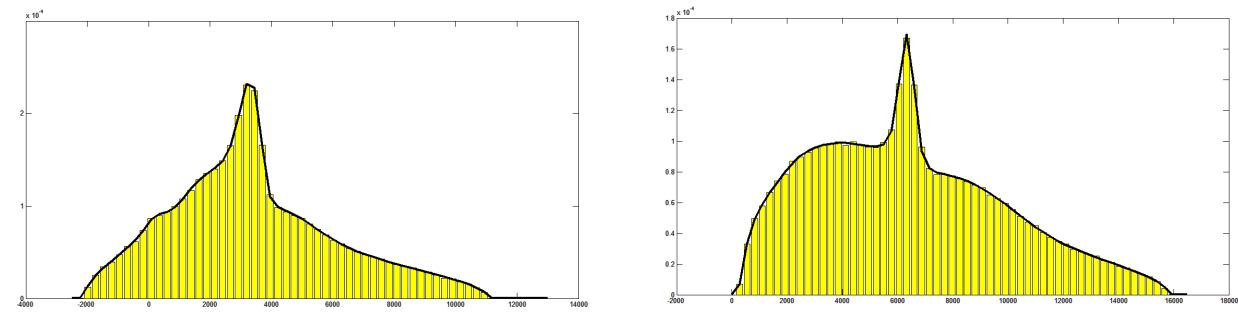

Figure 3. Spectral distribution of $\left(S_{2}^{\prime}+85 I_{2}\right)\left(S_{1}^{\prime}+40 I_{3}\right)$ (left) and $\left(S_{2}^{\prime}+85 I_{2}\right)\left(S_{1}^{\prime}+75 I_{3}\right)$ (right) - random matrix simulations (histogram) compared with numerically calculated density using our method.

3.2. Distribution of $d c d+d^{2} c d^{2}$. We consider now a special case of the problem from the Introduction, namely of finding the distribution of $d c d+d^{2} c d^{2}$, where $c$ and $d$ are free from one another. As noted in the Introduction

$$
\left(\begin{array}{cc}
d c d+d^{2} c d^{2} & 0 \\
0 & 0
\end{array}\right)=\left(\begin{array}{cc}
d & d^{2} \\
0 & 0
\end{array}\right)\left(\begin{array}{cc}
c & 0 \\
0 & c
\end{array}\right)\left(\begin{array}{cc}
d & 0 \\
d^{2} & 0
\end{array}\right)
$$


has the same distribution as

$$
\left(\begin{array}{ll}
c & 0 \\
0 & c
\end{array}\right)\left(\begin{array}{ll}
d^{2} & d^{3} \\
d^{3} & d^{4}
\end{array}\right)
$$

Now, since $c$ and $d$ are free, the problem of calculating the distribution of $d c d+d^{2} c d^{2}$ has been transformed into one which can be solved numerically using the iterative method proposed in this paper. It is crucial to note that since $c$ and $d$ are free, we have that $\left(\begin{array}{ll}d^{2} & d^{3} \\ d^{3} & d^{4}\end{array}\right)$ and $\left(\begin{array}{ll}c & 0 \\ 0 & c\end{array}\right)$ are free over the matrices $M_{2}(\mathbb{C})$.

In order to apply our iterative method from Section 2, we need as input the operator-valued Cauchy transforms (or the $h$ transforms) of the matrices $x$ and $y$, where

$$
x=\left(\begin{array}{cc}
c & 0 \\
0 & c
\end{array}\right) \text { and } y=\left(\begin{array}{ll}
d^{2} & d^{3} \\
d^{3} & d^{4}
\end{array}\right) .
$$

For instance, we have that

$$
G_{x}(b)=\mathbb{E}\left[(b-x)^{-1}\right]=\mathbb{E}\left[\frac{1}{\left(b_{11}-c\right)\left(b_{22}-c\right)-b_{12} b_{21}}\left(\begin{array}{cc}
b_{22}-c & -b_{12} \\
-b_{21} & b_{11}-c
\end{array}\right)\right]
$$

and a similar formula for $G_{y}$. (Note that the entries of the $2 \times 2$ matrix $b-x$ commute and thus the usual formula for matrix inversion applies.) Thus we need to be able to calculate quantities like

$$
\tau\left[\left[\left(b_{11}-c\right)\left(b_{22}-c\right)-b_{12} b_{21}\right]^{-1}\left(b_{22}-c\right)\right]
$$

in order to calculate $G_{x}(b)$.

For instance, if we assume here that $c$ and $d$ are both discretely distributed, such expressions can be readily written down in analytic forms. Consider the concrete example where $c$ is uniformly distributed with discrete support $\{0.4,0.7,1,1.3,1.5,1.7\}$ and $d$ is uniformly distributed with discrete support $\{0.5,1,1.5,2,2.5,3\}$. In this case, the distribution of $d c d+d^{2} c d^{2}$ computed using the iterative method proposed in this paper is shown and compared to histograms in Figure 4.

We also compute the distribution of $d c d+d^{2} c d^{2}$ where $d$ is uniformly distributed over support $\{0.4,0.7,1,1.3,1.5,1.7\}$ and $c$ is a shifted semi-circular element (Figure (4).
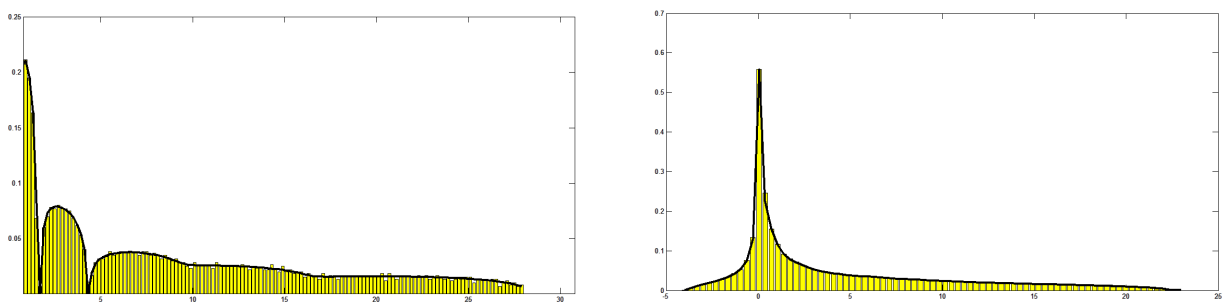

Figure 4. Comparison of distribution of $d c d+d^{2} c d^{2}$ where c is uniformly distributed over $\{0.4,0.7,1,1.3,1.5,1.7\}$ and $\mathrm{d}$ is uniformly distributed over $\{0.5,1,1.5,2,2.5,3\}$ (left) and where $\mathrm{c}$ is a shifted semi-circle and $\mathrm{d}$ is uniformly distributed over $\{0.4,0.7,1,1.3,1.5,1.7\}$ (right). 


\section{REFERENCES}

[1] N. I. Akhieser, The classical moment problem and some related questions in analysis. Translated by N. Kemmer. Hafner Publishing Co., New York (1965).

[2] S. Belinschi and H. Bercovici, A new approach to subordination results in free probability. Journal d'Analyse Mathematique, 101 (2007), 357-365.

[3] S. Belinschi, T. Mai, and R. Speicher: work in progress.

[4] S. Belinschi, M. Popa, and V. Vinnikov, Infinite divisibility and a non-commutative Booleanto-free Bercovici-Pata bijection. Journal of Functional Analysis, 262 (2012), Issue 1, 94-123.

[5] F. Benaych-Georges, Rectangular random matrices, related free entropy and free Fisher's information. Journal of Operator Theory, 62, no. 2 (2009), 371-419.

[6] H. Bercovici and V. Pata. Stable laws and domains of attraction in free probability theory. With an appendix by P. Biane. Ann. of Math. (2) 149 (1999), no. 3, 1023-1060.

[7] G. Blower Random Matrices: High Dimensional Phenomena Cambridge University Press, 2009.

[8] H. Bercovici and D. Voiculescu Free convolutions of measures with unbounded support. Indiana Univ. Math. J. 42 (1993), no. 3, 733-773.

[9] P. Biane, Processes with free increments. Math. Z. 227 (1998), 143-174.

[10] W. Bryc, A. Dembo and T. Jiang. Spectral measure of large random Hankel, Markov and Toeplitz matrices. Ann. Probab., 34 (1): 1-38, 2006.

[11] S. Dineen, Complex Analysis in Locally Convex Spaces. Notas de Matemática (83), North Holland Mathematics Studies 57, North Holland Publishing Company, 1981.

[12] , The Schwarz lemma. Oxford University Press, 1989.

[13] K. Dykema, On the S-transform over a Banach algebra. J. Funct. Anal. 231(2006) no.1, 90-110.

[14] W. Helton, R. Rashidi Far, and R. Speicher, Operator-valued semicircular elements: solving a quadratic matrix equation with positivity constraints. Int. Math. Res. Not. IMRN (2007), no. 22 , Art. ID rnm086, 15.

[15] A. Nica and R. Speicher, Lectures on the combinatorics of free probability. Cambridge University Press (2006).

[16] Ch. Pommerenke Boundary behaviour of conformal maps. Grundlehren der Mathematischen Wissenschaften [Fundamental Principles of Mathematical Sciences], Vol 299, Springer-Verlag, Berlin, 1992

[17] R. Rashidi Far, T. Oraby, W. Bryc, R. Speicher: On slow-fading MIMO systems with nonseparable correlation. IEEE Trans. Inf. Theory, 54 (2008), 544-553.

[18] D. Shlyakhtenko, Random Gaussian band matrices and freeness with amalgamation. Internat. Math. Res. Notices (1996), no. 20, 1013-1025. MR 1422374 (97j:46070)

[19] _ A-valued semicircular systems. J. Funct. Anal. 166 (1999), no. 1, 1-47. MR MR1704661 (2000j:46124)

[20] R. Speicher. Combinatorial theory of the free product with amalgamation and operator-valued free probability theory. Memmoir of the AMS, 627 (1998).

[21] R. Speicher, C. Vargas. Free deterministic equivalents, rectangular random matrix models and operator-valued free probability. Random Matrices: Theory and Applications 1 No.2 (2012).

[22] B. Szökefalvi-Nagy, C. Foias, H. Bercovici and L. Kérchy, Harmonic Analysis of Operators on Hilbert Space. Second Edition, Springer 2010.

[23] A. Taylor, Analysis in complex Banach spaces. Bull. Amer. Math. Soc. 49, 1943, 652-669.

[24] D. Voiculescu, Symmetries of some reduced free product $C^{*}$-algebras, Operator algebras and their connections with topology and ergodic theory (Buşteni, 1983), Lecture Notes in Math., 1132, Springer, Berlin, (1985), 556-588. MR MR799593 (87d:46075)

[25] _ Addition of certain non-commutative random variables. J. Funct. Anal., 66 (1986), $323-346$

[26] Multiplication of certain noncommuting random variables. J. Operator Theory 18(1987), 223-235.

[27] _ Limit laws for random matrices and free products. Invent. Math.,104 (1991), 201220.

[28] theory. I. Comm. Math. Phys. 155 (1993), 411-440. 
[29] - Operations on certain non-commutative operator-valued random variables. Astérisque (1995), 232, 243-275, Recent advances in operator algebras (Orléans, 1992). MR 1372537 (97b:46081)

[30] , The coalgebra of the free difference quotient and free probability. Internat. Math. Res. Notices (2000), 2, 79-106. MR MR1744647 (2001d:46096)

[31] _ Analytic subordination consequences of free Markovianity. Indiana Univ. Math. J. 51 (2002), 1161-1166.

[32] D. Voiculescu, K. Dykema and A. Nica. Free random variables. CRM Monograph Series, 1, AMS, 1992.

Department of Mathematics \& Statistics, Queen's University, and Institute of Mathematics "Simion Stollow" of the Romanian Academy; Department of Mathematics and Statistics, Queen's University, Jeffrey Hall, Kingston, ON K7L 3N6 CANADA

E-mail address: sbelinsch@mast.queensu.ca

Universität des SaArlandes, FR 6.1-Mathematik, Postfach 151150, 66041 SaArbrüCken, GERMANY

E-mail address: speicher@math.uni-sb.de

Department of Mathematics \& Statistics, Queen's University; Department of Mathematics and Statistics, Queen's University, Jeffrey Hall, Kingston, ON K7L 3N6 CANADA

Universität des SaArlandes, FR 6.1-Mathematik, Postfach 151150, 66041 SaArbrücken, GERMANY

E-mail address: carlos@math.uni-sb.de 